\title{
Le récit de Lessa. Des cultes de possession aux Églises
} pentecôtistes

In: L'Homme, 1998, tome 38 n¹48. pp. 119-137.

Citer ce document / Cite this document :

Boyer Véronique. Le récit de Lessa. Des cultes de possession aux Églises pentecôtistes. In: L'Homme, 1998 , tome 38 n¹48. pp. 119-137.

doi : $10.3406 /$ hom.1998.370579

http://www.persee.fr/web/revues/home/prescript/article/hom_0439-4216_1998_num_38_148_370579 


\title{
Le récit de Lessa Des cultes de possession aux Églises pentecôtistes
}

\author{
Véronique Boyer
}

\footnotetext{
O
}

utre les Églises introduites par des missionnaires étrangers pendant la première moitié du siècle, le pentecôtisme brésilien compte des Églises proprement nationales ${ }^{1}$, fondées au cours des trois dernières décennies dans le sud-est du pays et en plein essor depuis les années 1970-1980. À Belém, capitale d'un des États de l'Amazonie brésilienne (l'État de Pará), comme dans le reste du Brésil, le prosélytisme acharné des Églises leur permet de gagner à la "vraie croyance " des catholiques convaincus de l'« idolâtrie" de leurs pratiques antérieures et d'anciens médiums des cultes de possession dits afro-brésiliens plus farouchement encore associés au diable. Du fait d'une implantation sociologique semblable (les secteurs pauvres de la population), la rivalité opposant le pentecôtisme à ces deux mouvements, qui ont longtemps été considérés par les intellectuels comme les seules expressions légitimes de la religiosité populaire, se manifeste dans un climat souvent très tendu.

Les Eglises évangéliques défendent une conception dualiste du monde où le Bien qu'elles représentent s'oppose au Mal que les autres systèmes servent, et à partir de laquelle ceux-ci se voient attribuer des positions dans le champ religieux. Autour de ce centre évangélique du Bien, se répartissent catholiques, spirites et médiums, qui représentent autant de versions plus ou moins inquiétantes du Mal. Cet espace du Bien, conçu comme une place forte constamment menacée mais sévèrement défendue, offrirait confort et sécurité aux élus de Dieu contre les agressions du monde. Le jeu de la concurrence porte ainsi les Églises à insister sur l'idée d'un ensemble fini, protégé par des limites bien établies et un personnel vigilant.

Dans le discours pentecôtiste officiel où la hargne le dispute à l'incompréhension, les cultes de possession sont tenus pour l'une des formes religieuses les plus diaboliques. Les médiums des cultes, appelés aussi fils-de-saint, sont ainsi accu-

1. La première de ces Églises fut l'Assemblée de Dieu implantée tout d'abord dans le nord du pays en 1911. 
sés d'être possédés et utilisés par d'innombrables démons qui répandraient le Mal sur terre par leur entremise. Au nom d'une mission de salut des âmes, les pentecôtistes tentent alors de convaincre les médiums de rejoindre leurs rangs en s'attaquant au sens que revêt la possession pour ces derniers. Plutôt que de rejeter et de condamner purement et simplement la transe possessive, les Églises proposent de la réinterpréter dans leur langage, en faisant de cette pratique cultuelle un phénomène démoniaque qui doit être traité par l'exorcisme. Ainsi, les Églises assurent les médiums vacillants de leur capacité à donner du sens à la possession à partir d'une nouvelle grille de lecture.

Car, si pour les fils-de-saint la possession constitue la confirmation de leur alliance avec les êtres invisibles qui les chevauchent dans les lieux de culte ou terreiros, elle est interprétée par les pentecôtistes comme une preuve de l'ascendant du diable sur les hommes. La redéfinition du sens de la possession et de sa légitimité est toujours un thème sensible dans le récit des anciens fils-de-saint passés au pentecôtisme. Après avoir consacré beaucoup de temps et d'énergie à rendre incontestable par leur entourage le lien qui les unit aux esprits, ils devront, pour devenir pentecôtistes, se soumettre à un processus inverse dont l'objectif, à travers les séances d'exorcisme, est de rompre cette même relation.

Cependant, si le sens attribué aux éléments symboliques et rituels change nettement quand le narrateur appartient aux cultes de possession ou à une Église pentecôtiste, la structure du récit des itinéraires religieux ne semble guère différer. Il est vrai que reviennent fréquemment dans la bouche des pentecôtistes des expressions absentes du récit des médiums, telles que "depuis que j'ai la connaissance [biblique], je sais... " ou " je n'aime pas raconter tout ce qui est arrivé ", qui tendent à suggérer la rupture sociale et personnelle effective opérée lors de l'adhésion à la "vraie croyance " et le rejet des expériences du passé. Mais ces affirmations réitérées contrastent fortement avec une parole souvent prolixe sur cette " mauvaise vie " antérieure qui cherche à justifier l'appartenance religieuse présente.

Les divers éléments du récit acquièrent pour leur auteur d'autres significations compte tenu du nouveau contexte discursif. On doit à ce point noter que l'effet produit par la narration induit chez l'auditeur le sentiment d'un ajout de sens à l'interprétation première des événements plutôt que celui de sa négation à proprement parler ${ }^{2}$. Par exemple, si les esprits-électeurs des médiums, leurs caboclos, sont rétrospectivement assimilés aux démons par les nouveaux pentecôtistes, ils conservent globalement leurs attributs initiaux, que ce soit leur nom ou les traits de comportement qui leur sont prêtés dans les terreiros lors de la possession. Pour une large part, les médiums des terreiros ne désavoueraient d'ailleurs pas la lecture que des pentecôtistes convaincus font des événements passés comme une lutte sans merci menée par des êtres invisibles voulant soumettre les hommes. La modification d'attitude parallèle au changement d'appartenance religieuse (de cultes réputés pour leur tolérance ou leur indifférence à l'égard d'autres pratiques religieuses se tournant vers un mouvement où tout ce qui est différent est rejeté

2. Christiane Bougerol $(1993$ : 101) analyse un phénomène analogue à la Guadeloupe où les gens ne vivent pas leur changement d'appartenance religieuse comme un "passage de l'erreur à la vérité ". 
du côté du démon) n'empêche pas une remarquable continuité dans le traitement de certains thèmes.

La présentation d'une histoire de vie me permettra de souligner les moments de la recomposition de l'univers personnel et social, et de restituer ainsi à des itinéraires religieux leur dimension de trajectoires sociales. La conduite de ce récit indique également comment, à partir d'une trame par ailleurs parfaitement stéréotypée, sont insérées de nombreuses variations qui témoignent de l'ingéniosité de la narratrice pour articuler ses options passées et présentes, sans que jamais affleure une contradiction insurmontable. Car les contradictions, loin de constituer un obstacle à la compréhension des événements, sont au contraire porteuses de sens.

Lessa, qui en 1995 (date du terrain) était âgée de trente-neuf ans, a passé plus d'une douzaine d'années dans les cultes de possession, tout d'abord comme médium dans le terreiro d'un chef de culte, puis, une fois libérée de cette tutelle, est devenue mère-de-saint. Mais depuis environ quatre ans, elle a quitté l'univers des cultes de possession pour celui des Églises pentecôtistes. Après l'exorcisme de ses démons par un pasteur, elle fréquente assidûment l'Église quadrangulaire fondée en 1922 aux États-Unis par une femme, Aimee Semple McPherson, arrivée dans le sud du Brésil dans les années 40 puis à Belém dans les années 70 . Lessa est devenue un membre important de la congrégation de son quartier : elle est à présent à la tête d'un groupe d'une cinquantaine de femmes de tous âges qui se réunit plusieurs fois par semaine pour discuter, prier et visiter celles qui ne peuvent se déplacer. Cette fonction enviable de présidente du cercle des femmes lui vaut d'être fréquemment sollicitée à son domicile par des épouses ou des mères en détresse. Bien connue de ses voisins pentecôtistes et des autres, elle bénéficie d'une position sociale bien assurée dans son entourage.

\section{Le chemin vers les cultes de possession}

ou se donner au diable

Lessa réside dans un quartier distant du centre de Belém et d'accès relativement difficile par les transports en commun. La construction de la Ville Nouvelle, divisée en sections administratives au fur et à mesure de son expansion (celle où habite Lessa est la VIe, l'une des dernières), a obéi à une planification rigoureuse : les rues goudronnées se recoupent à angle droit, les maisons y sont pour la majorité en dur mais il y a peu de commerces et encore moins de lieux de distraction. La réputation de violence et de marginalité, associée à d'autres quartiers moins pourvus en équipements urbains où loge une population démunie, touche également la Ville Nouvelle et ses habitants. La modeste maison que Lessa occupe avec son mari âgé de cinquante-trois ans et leur fille de onze ans n'abrite pas seulement la famille nucléaire. Le groupe domestique s'est un temps ouvert à deux de ses nièces, privilégiant comme dans la plupart des cas l'élargissement de la maisonnée du côté matrilatéral, et la plus jeune d'entre elles habite encore aujourd'hui avec Lessa.

C'est dans l'enfance que Lessa commence à souffrir de troubles qui perturbent profondément sa vie sociale. L'existence qu'elle mène dans la petite ville de l'in- 
térieur amazonien, Obidos, où elle est née, sa participation aux temps forts de la sociabilité locale (les processions catholiques et les anniversaires de ses amis) ne comblent pas le vide, l'angoisse qu'elle ressent. À l'âge de quinze ans, tenaillée par une peur croissante inspirée par l'univers étriqué de sa ville natale et désireuse de conquérir son indépendance, elle attend l'occasion propice pour partir. Celle-ci se présente sous la forme d'une proposition de travail que lui fait un couple en partance pour Belém.

Ses nouveaux maîtres se montrent assez généreux par rapport au modèle habituel régissant les relations entre patrons et employée domestique : traitant Lessa comme une fille de la maison, dit-elle, ils l'envoient à l'école. Elle ne profitera malheureusement pas autant qu'elle l'aurait souhaité de cette chance car, "sur le tableau noir, les lettres s'embrouillent devant ses yeux ». Ces difficultés expliquent, selon Lessa, l'interruption de ses études primaires. Mais elle obtient par la suite un diplôme de coiffure dans un cours professionnel.

La vie citadine ne tient pas ses promesses : l'apprentissage et le travail se révèlent des contraintes encore plus pesantes que celles entravant Lessa à Obidos. C'est tout du moins ce que son récit laisse entendre. Loin de disparaître, son mal se précise et son expression emprunte aux cultes de possession un langage dont elle prend connaissance lors de discussions avec des bonnes du quartier : le sentiment d'oppression, la perte de sommeil, la peur et les crises de nerfs seraient la conséquence de rêves éveillés où des hommes l'accompagnent dans la rue et lui ordonnent de se jeter sous un autobus ou dans un puits. Ses patrons lui conseillent en vain de se confesser au prêtre catholique.

"Je faisais des rêves dans lesquels j'étais prise et emmenée au fond de l'eau comme si j'avais plongé. Des rêves horribles, des cauchemars. Je me suis sentie opprimée ${ }^{3}$ depuis toute petite. Je voyais des ombres, des hommes, j'entendais des voix qui tourmentaient ma vie. Ces voix ne me disaient jamais de bonnes choses. Elles disaient seulement des choses terribles."

" J'ai même été hospitalisée. Quand j'étais à l'hôpital, j'étais comme anesthésiée. Mais quand l'effet du médicament passait, quand je revenais, comment dire, à mon état normal, je m'enfuyais de l'hôpital, je prenais le premier taxi qui passait dans la rue. Parce que, pour moi, j'étais normale. Mais je pleurais au milieu de la rue, je n'arrivais pas à savoir où j'allais, je me perdais dans une rue connue, tout devenait différent à mes yeux, et je marchais en pleurant sans raison comme si quelqu'un m'avait battue. "

Lessa, désorientée et insatisfaite, fait une seconde tentative dans sa quête d'indépendance. Trouvant un emploi dans un salon de coiffure, elle quitte la maison de ses patrons pour habiter avec des cousins, originaires comme elle de Obidos. Cependant, les visions et les hallucinations auditives se multiplient. La nuit tombée, sur le chemin du retour, elle se sent persécutée par les propos moqueurs de femmes aux longs cheveux et poursuivie jusqu'à sa porte par des hommes invisibles aux autres êtres humains. Le récit de ses aventures se heurte en outre à l'incrédulité de ses cousins qui la traitent de folle lorsqu'elle se risque à leur confier

3. Le vocabulaire employé par Lessa est ici différent de celui des médiums des cultes qui au terme oprimida préferent celui de souffrante (sofrida) ou suffoquée (sufocada). 
que l'un de ces "hommes" coucherait avec elle. Lessa, sans jamais réussir à convaincre ses proches de sa bonne foi et à les rallier à sa version des événements, ne cessera pas pour autant d'affirmer sans relâche: "Je sais que je suis normale même si les autres ne me croient pas ; pourtant il faut bien reconnaître qu'un certain nombre de choses dans ma vie sont, elles, anormales."

Cette période se place sous le signe d'une double transgression au regard d'une définition de la féminité par l'incarnation des valeurs familiales et la proximité de l'univers domestique ${ }^{4}$. Lessa semble en effet adopter un comportement masculin. Elle consomme de l'alcool pour se "donner du courage " et surmonter " une angoisse, une tristesse et une amertume venues de je ne sais où ". Elle passe aussi le plus clair de son temps dans la rue qui est tenue pour un espace où les rencontres occasionnelles menacent de détourner les êtres humains de leurs obligations familiales. Chaque fois que l'oppression devient trop insupportable, elle descend de l'autobus pour se réfugier dans un taxi, espérant trouver en la personne du conducteur une oreille attentive. Lessa a vingt-deux ans quand elle rencontre enfin un chauffeur de taxi, Pedro, qui s'intéresse assez à son histoire pour garer son véhicule sur l'une des places principales de Belém. Lorsqu'elle a fini de s'épancher, il l'engage, affirme-t-elle, à chercher une "maison" (entendons un lieu de culte) pour découvrir l'origine des " perturbations» dont elle souffre. Pedro, non content d'ajouter foi en ses propos, lui aurait donc proposé de la conduire chez un chef de culte de sa connaissance, une "femme qui travaille".

La version de cet épisode retenue ici par Lessa est bien différente de ce que les recoupements d'informations ont permis d'établir. En fait le terreiro où elle se rend lui a été indiqué par une voisine, également originaire de Obidos. La substitution d'un personnage féminin par un personnage masculin dans la position $\mathrm{d}^{\prime}$ annonciateur ${ }^{5}$ n'est pas anodine ; elle suggère que l'histoire contée par Lessa est loin d'avoir trouvé son dénouement. Car dans la narration des femmes médiums, le premier contact avec les cultes de possession est systématiquement un événement féminin, et l'homme apparaît invariablement comme le personnage qui contrarie leur vocation, celui qui confond les caboclos avec des démons et qu'il leur faudra soumettre quitte à le chasser du toit familial ${ }^{6}$. Le rôle que Lessa attribue à Pedro rompt ainsi singulièrement avec celui que les femmes médiums réservent dans le récit à leur compagnon. Le thème de la complicité féminine et celui de la lutte des femmes contre leurs hommes pour conquérir un espace indépendant apparaissent plus tard, lorsque Lessa évoque son adhésion au pentecôtisme et que Pedro se voit doté d'un rôle plus conforme aux critiques féminines.

Cette femme, qui insiste volontiers sur son ignorance à l'époque en matière de cultes de possession, découvre alors l'alternative à laquelle tous les médiums ont un jour été confrontés. Mère Diane, dont le lieu de culte est situé sur une grande

4. Sur les rôles et sphères dévolus aux hommes et aux femmes dans le Brésil urbain, voir par exemple Claudia Fonseca (1984) et Alba Zaluar (1982).

5. Voir Jeanne Favret-Saada (1985).

6. Sur ce point, voir Véronique Boyer (1993). 
avenue à la sortie de la ville, émet un diagnostic de "médiumnité en train d'éclore (desabrochada) et de début de folie ". Or le destin de " folie " ne peut être conjuré que par la soumission aux êtres invisibles et l'accomplissement d'une mission. Lessa accepte cette interprétation des choses anormales de son existence en termes d'une élection par des esprits désireux d'user de sa personne, à travers la possession, pour intervenir dans la vie des hommes et aider de leurs conseils ceux qui souffrent.

Pedro est très présent pendant toute cette période bien que, toujours selon Lessa, il n'a jamais beaucoup aimé les cultes : il l'attend à la sortie de son travail, la conduit au terreiro et achète, avec ses propres deniers, le matériel réclamé par la mère-de-saint pour faire une offrande aux esprits, les apaiser en montrant la bonne volonté de Lessa.

"Quand je suis arrivée là, la femme m’a demandé de m'habiller avec des vêtements blancs, et il les a achetés tout de suite. Je suis restée là. Alors la femme lui a demandé beaucoup de choses. Elle a demandé des pigeons, pour tuer les trois pigeons sur ma tête et recueillir ce sang. Les médiums ont fait tellement de choses sur ma tête, un groupe de femmes dansait, et le tambour résonnait par-ci et par-là. »

Du point de vue pentecôtiste aujourd'hui adopté par Lessa, cette offrande n'a cependant pas simplement consacré son alliance avec les caboclos. Elle a en réalité scellé un pacte avec le diable, pacte concrétisé par l'expérience, nouvelle pour Lessa, de la possession. Car elle en est à présent certaine, céder à la pression des caboclos, ou plutôt renoncer à leur résister, c'est s'en remettre au diable.

"Ça a été ma perdition, réellement. Ma perdition parce que j'avais eu une vie très difficile, mais à ce moment je me livrais encore plus au diable. Jusque-là quand j'avais peur, et que je courais et m'enfuyais, je ne m’étais pas totalement donnée à lui (entrega). Mais dès le moment où j'ai accepté d'aller là, j'étais vraiment dans la main de Satan. À partir de là, j'ai été utilisée. Je suis arrivée là et j'ai été possédée aussi. On aurait dit que je tombais au milieu de fourmis, l'esprit malin du diable est descendu dans ma tête, c'est-à-dire qu'il a utilisé mon corps. Alors je lui ai appartenu." "

Pour recevoir plus facilement les soins que nécessite son état, Lessa loue avec Pedro une petite maison dans l'impasse même où réside mère Diane. Sous la houlette de cette dernière, la voilà qui pendant sept ans fait son apprentissage de l'univers des cultes pour reconnaître et servir les esprits représentés par les autres médiums du terreiro. Elle incarne elle-même plusieurs caboclos (Erundina et Rompe-Mato) et un exu féminin (Maria Padilha). Assumant son rôle d'intermédiaire au mieux de ses possibilités, Lessa semble avoir enfin trouvé sa place et un certain calme, comme le montre, dit-elle, son sommeil retrouvé.

7. Les médiums des cultes reconnaissent plusieurs catégories d'esprits. Les caboclos qui forment le groupe le plus important se voient attribuer une nature multiple : ce sont des esprits de personnes décédées de mort violente mais aussi les esprits d'êtres humains disparus (en ce cas leur corps n'aurait pas été retrouvé). Ils peuvent en outre se présenter sous une forme animale. Un caboclo pourrait également posséder les médiums en tant qu'exu, divinité souvent associée au diable de la tradition catholique. Je m'en tiens ici aux esprits mentionnés par Lessa. Sur les orixas (les divinités au nom africain), les pretosvelhos (esprits des anciens esclaves) et les crianças (esprit des enfants morts en bas âge) à Belém, voir V. Boyer (1993). 


\section{De médium à chef de culte :}

une dépendance chaque fois plus pesante

Dans ce terreiro, Lessa identifie le personnage de ses visions qui est censé abuser d'elle. Il s'agit du boto, un dauphin d'eau douce, illustre figure des mythes amazoniens, dont les organes génitaux entrent dans la composition de préparations magiques. Grand séducteur de femmes et surtout de vierges (Galvão 1976 : 68), le boto est parfois invoqué lors de la naissance d'un enfant illégitime. Il aurait la faculté de prendre une forme humaine, y compris celle du mari, pour leurrer des femmes qui deviendraient alors " anémiques ". Le sang attirant fortement le boto, il est fermement déconseillé à celles-ci de se baigner dans le fleuve pendant la période de menstrues ${ }^{8}$. L'origine de Lessa, l'hinterland amazonien où le personnage du boto a davantage d'épaisseur qu'en milieu urbain, explique très certainement qu'elle ait exploité, plus que d'autres médiums, le principe du polymorphisme des caboclos'. Car ces êtres invisibles, dont l'effigie est habituellement anthropomorphique, pourraient également se manifester aux hommes sous une forme animale.

Lessa, appliquant la grille de lecture des cultes urbains à un personnage issu du monde rural, se déclare seul objet du désir du boto. Celui-ci l'aurait choisie depuis toujours, il se comporterait avec elle comme un "compagnon" jaloux qui, n'acceptant pas l'intrusion d'une autre figure masculine, bien réelle celle-là, voudrait à tout prix chasser son concurrent, c'est-à-dire Pedro. La participation de Lessa aux terreiros lui permet de comprendre par la même occasion la signification des "voyages au fond de l'eau " où l'entraînait le boto: marque d'élection personnelle que la plupart des médiums exhibent également.

Cependant, contrairement aux filles-de-saint qui font de leur tourmenteur invisible un double dans la possession, le boto de Lessa est incarné par une autre femme, sœur de la mère-de-saint, chef de culte elle-même et " amie " de celui qui deviendra quelques années plus tard son mari ${ }^{10}$.

« Le boto descendait dans la tête d'une autre médium. Il me parlait, à moi et à mon mari : "C'est moi qui fais ça, disait-il parce que je suis son mari." Parce qu'en vérité il avait des relations avec moi. Il portait tous les jours les mêmes vêtements, il venait et avait des relations avec moi. Il venait comme une personne, avec un corps physique, il entrait. Je savais que la maison était fermée mais il entrait. Il venait comme un homme bien habillé, parce qu'on dit, parce quaujourd'hui j'ai la connaissance, je sais... ${ }^{11}$ Le boto utilisait le corps d'une autre médium pour se bagarrer avec mon mari. Il disait qu'il vivait avec moi depuis toute petite. C'est vrai, parce que depuis mon enfance, je le voyais avec moi mais je ne savais pas pourquoi, ni qui c'était. Il ne m’a jamais possédée. Il recherchait toujours une autre médium qu'il aimait, qu'il appelait fille, Maria, qui était la sœur de la mère-de-saint. Elle avait même un terreiro."

8. Les pêcheurs ne résisteraient pas non plus aux attraits du boto femelle (Wagley $1977: 234-236$ ).

9. Sur la constitution de ce personnage des cultes, voir V. Boyer (1993).

10. Le terme mari est utilisé pour désigner le conjoint que l'union soit légale ou non.

11. "Parce qu'on dit, parce qu'aujourd'hui j'ai la connaissance, je sais... ", l'expression est intéressante parce qu'elle montre le glissement d'une formule générale et diffuse, faisant état d'un dire coutumier des anciens, à une affirmation personnelle fondée sur un savoir présent et spécifique (celui de tout pentecôtiste) ainsi que sur une expérience individuelle qui renforce " en connaissance de cause "la première proposition. 
Lessa rencontre donc sur son chemin un obstacle de taille. Elle est confrontée à une femme, Maria, qui, du fait de son ancienneté et de son "lien de sang " ${ }^{12}$ avec la mère-de-saint, occupe une position supérieure à elle dans le terreiro et, qui plus est, est elle-même chef de culte. Car une néophyte en matière de caboclo ne peut se mesurer à la propriétaire d'un terreiro. Dès lors Lessa, qui ne parvient pas à s'approprier le personnage du boto et à faire ainsi reconnaître sa compétence en tant que médium, connaît une recrudescence de ses troubles. D'autant que cette concurrence entre spécialistes religieux dissimule à peine la rivalité entre les deux femmes pour le même homme. Lessa reproche en effet à Maria de monopoliser son mari, en lui demandant de la conduire à l'école d'infirmière. Aussi, quand Maria, s'abritant derrière la jalousie supposée du boto, affirme que « le mari de Lessa doit s'éloigner » de celle-ci, les langues se délient et insinuent qu'il s'agit moins d'évincer Pedro que d'écarter Lessa.

Cette dernière finira cependant par l'emporter. Se rangeant à ses côtés, Pedro se déclare las de "se battre contre une personne qu'il ne voit pas " et il prie la mère-de-saint de procéder à un sacrifice pour calmer le boto. Cette offrande, déposée sur la plage comme il se doit pour un esprit de l'eau, sera suivie d'une seconde, cette fois-ci en l'honneur des exus et placée au croisement de deux rues, avant que le boto ne cesse d'importuner Pedro. Retournant à son invisibilité d'antan, ce personnage persiste à persécuter la seule Lessa. C'est ainsi que sous l'influence du boto elle aurait enfreint les normes habituelles de comportement et adopté des attitudes inconvenantes.

On observe ici un premier écart de langage par rapport au vocabulaire des cultes. Car Lessa parle de cette emprise du boto sur sa personne comme d'un encosto, terme désignant habituellement la relation néfaste avec l'âme d'un mort récemment décédé dans des circonstances violentes. Refusant le brusque changement de condition, ce mort, qui peut être un parent ou un proche de la personne tourmentée, chercherait à vivre coûte que coûte par le truchement d'un autre, en "s'accrochant à lui " par un encosto. Or ce terme ne saurait qualifier le contact avec le boto car cet être non humain est par définition un esprit «bon pour la possession ". Les symptômes présentés par Lessa seraient, pour les filsde-saint, les signes somme toute assez communs de l'intérêt pressant d'un caboclo à l'égard d'un médium.

Lessa, ayant donc réussi à garder son mari, doit en payer le prix : le départ du terreiro de mère Diane qu'elle explique dans des termes proches de ceux des médiums des cultes. Ainsi, le "travail » que lui imposent les caboclos dont elle dépend pour ne pas retomber dans la maladie, le sang des animaux sacrifiés qu'ils l'obligent à boire et les "perturbation " que son foyer continue à souffrir sont les éléments qui cachent sa déroute professionnelle. Toutefois, tout au long de cette énumération, elle ne manque pas de s'attribuer quelques-unes des qualités qui sont l'apanage des médiums compétents : la perte totale de conscience lors de la possession, l'absence de maîtrise de son corps et les gestes " répugants » effectués

12. Les relations entre consanguins sont censées être plus stables et plus fortes que les liens noués par l'alliance. À propos de l'idéologie de la consanguinité, voir C. Fonseca (1988). 
par le fils-de-saint sous l'emprise des êtres invisibles. Bien que pentecôtiste au moment du récit, Lessa ne suggère à aucun moment que les médiums se placent de leur propre chef du côté du mal et elle insiste sur le fait que l'esprit est différent de l'être humain qu'il possède ${ }^{13}$. En prenant soin de distinguer sa compétence en matière de culte de l'objectif - nuisible, de son point de vue actuel - que les caboclos poursuivent, elle peut revendiquer son appartenance présente au pentecôtisme sans rejeter les interprétations antérieures de ses troubles. Ses talents s'exerçaient simplement dans un lieu inadéquat, et ils étaient mis au service d'une puissance impropre.

Elle décide alors de tenter sa chance en fondant un lieu de culte qui lui appartienne en propre. Le pas n'est pas facile à franchir et nombreux sont les médiums pour lesquels cela reste à l'état de projet. Les difficultés financières auxquelles se heurte Lessa sont d'autant plus importantes que, l'employeur de Pedro ayant fait faillite, son mari se retrouve au chômage. À force d'économies, ils réussissent néanmoins à réunir une somme suffisante pour acheter la petite maison de la Ville Nouvelle où ils résident aujourd'hui. Contre l'avis de sa mère-de-saint, jalouse de ses médiums comme toutes ses concurrentes, Lessa installe dans son nouvel environnement les statues de ses caboclos, offertes par des consultants reconnaissants, qui étaient jusque-là chez mère Diane. À trente-deux ans, la voilà chef de culte.

Tout en exerçant son métier de coiffeuse au domicile conjugal, elle commence à répondre à des demandes de services religieux. Son entreprise est dans un premier temps couronnée de succès. Ses exploits de possédée lui sont rapportés par ses proches et des clients satisfaits lui prouvent leur gratitude par des cadeaux aussi divers que de l'électroménager ou des bijoux fantaisie. Pourtant, elle renonce bientôt à poursuivre cette carrière religieuse. Pedro a-t-il pris ombrage des revenus de sa femme, devenus plus importants que les siens, et se sent-il dépossédé de son rôle de pourvoyeur du groupe domestique comme bon nombre des compagnons de ces femmes médiums? Ou bien Lessa se montre-t-elle une mère-de-saint trop médiocre pour se constituer une clientèle fidèle?

Elle ne parle que de cette "vieille sensation de vide intérieur " qui, loin d'être apaisée par son obéissance aux êtres invisibles, gagne chaque fois plus d'ampleur. Son malaise est en outre aggravé par une nouvelle rivalité entre femmes. Le besoin qu'elle ressent d'expliquer cet épisode la conduit à dépeindre Pedro sous un jour nouveau, non plus comme un guide protecteur mais comme un homme semblable à tous les autres : coureur de jupons invétéré et irresponsable. Avant de la suivre sur le chemin du pentecôtisme, son mari aurait en effet souffert d'une

13. Aucune idée de faute personnelle n'affleure tout au long du récit de Lessa qui affirmera en revendiquant son appartenance pentecôtiste : "L'ennemi était en moi, dans ma personne, mais ce n'érait pas vraiment moi. " Les pentecôtistes, tout comme les médiums des cultes, écartent les notions de péché et de culpabilité. Leur interprétation du malheur évoque davantage le registre de la relation persécutive (voir András Zempléni 1975). Loorigine des troubles est toujours le fait d'un élément allogène (esprit ou démon), bien distinct de la personne du possédé, même si les médiums des cultes y voient le signe d'une alliance possible avec les caboclos alors que, pour les pentecôtistes, il s'agit d'une agression du démon contre laquelle il convient de se défendre. 
"maladie " courante chez les hommes qui les pousse à accumuler les conquêtes féminines en ignorant les conséquences de leurs actes.

Lessa doit précisément essuyer la colère de l'une de ces femmes abandonnées, avec laquelle Pedro a vécu dix-huit ans et dont il a eu six enfants. La nouvelle du mariage de Lessa et Pedro, circulant de bouche à oreille, aurait éveillé le courroux de cette ancienne compagne. Prédisant à Lessa les pires choses, dont l'absence d'enfantement, la femme aurait usé de magie noire pour lui nuire, attaquant en particulier sa santé. Lessa, effrayée par les coqs noirs sacrifiés et les excréments humains déposés sur le pas de sa porte, serait allée consulter un chef de culte de ses amis. Mais elle refusera toujours, à l'étonnement de celui-ci note-t-elle, de retourner le charme contre son adversaire.

Aujourd'hui, de la place qu'elle occupe parmi les "vrais croyants", elle interprète sa réticence d'alors comme la preuve d'une autre élection qui, en raison de son antériorité, finira par supplanter celle par les caboclos. Car, avant même qu'elle ait reçu les premiers signes de l'intérêt des caboclos pour sa personne, quand elle était encore dans le "ventre de sa mère ", Jésus l'aurait couverte de son attention bienveillante. Il est remarquable que, dans le récit, cette substitution s'opère en continuité avec les représentations des médiums concernant leur contact avec les esprits avant même leur naissance. Ce remplacement effectué, ce sera au nom de Jésus qu'elle dit conjurer les attaques de sorcellerie dont elle est victime.

La fureur de Lessa se retourne contre les êtres invisibles. Un jour de colère elle jette les statues qui les représentent au fond du jardin. La punition des caboclos, à la mesure de son geste irrévérencieux, atteint sa fille de quatre ans (en 1981) qui, sans raison apparente, se met à sautiller dans toute la maison en poussant des cris à fendre l'âme. Les statues réintègrent aussitôt l'espace domestique. Mais Lessa ne renonce pas pour autant à en finir avec une dépendance devenue trop pesante. Elle confectionne un rideau à l'aide duquel elle isole les statues du reste de la maison, en priant Dieu de la délivrer de ces «choses", de ces "cochonneries". Cette initiative qui contrarie implicitement les injonctions des esprits aurait davantage effrayé son mari qu'elle-même. Quand elle soulève le rideau pour faire son ménage, la " haine des caboclos" qu'elle perçoit sur le visage grimaçant des statues de plâtre, loin de l'atterrer, provoque une réaction violente de sa part que résume une promesse de vengeance : "un jour vous me le paierez". Éloignée physiquement des lieux de culte et tentée par le spiritisme ${ }^{14}$, Lessa n'a toutefois pas encore rompu toutes ses attaches avec l'univers des terreiros.

\section{De médium à croyante : la libération}

Comme le dit Lessa, sa rencontre avec les croyants aurait été le fait du hasard, un jour où elle décide d'inscrire sa fille à un cours de natation. Mais cette fois-ci, la rencontre se déroule dans le cadre habituel des relations féminines. C'est en effet d'une femme qu'elle reçoit le message évangélique, et pas de n'importe laquelle : il s'agit d'une ancienne fille-de-saint, initiée dans l'État

14. Elle fréquentera également la secte orientaliste Seicho-no-ié. 
voisin du Maranhāo15, qui jouissait d'une excellente réputation parmi les médiums des cultes.

Alors que Lessa accompagne sa fille à la piscine, elle remarque un visage familier parmi les cuisinières du club, celui de Cecilia, qu'elle avait connue dans un terreiro et perdue de vue depuis longtemps. Après de chaleureuses retrouvailles, cette dernière s'enquiert de la "religion " de Lessa, proclame bien haut son appartenance au pentecôtisme et à l'Église quadrangulaire, puis l'incite " à se donner à son tour à Jésus ». Cependant Cecilia prévient Lessa que la lutte sera rude car « le diable est encore plus révolté contre les médiums qui quittent les terreiros. Il m’a pris deux enfants alors toi qui n'as qu'une fille... " Lessa est profondément touchée par cette conversation, moins d'ailleurs par le message délivré que par la personnalité et la position occupée précédemment par son interlocutrice dans le milieu des cultes. Si cette fille-de-saint renommée a pu rejoindre les pentecôtistes jugés intolérants et austères, pourquoi ne trouverait-elle pas non plus un réconfort parmi eux ? Faisant fi de sa piètre opinion des croyants et craignant pour la santé de sa fille, Lessa s'engage à se rendre sous peu au temple de l'Église quadrangulaire. Mais avant qu'elle ne tienne sa promesse, il lui faudra vaincre ses propres hésitations et l'opposition de Pedro.

Le jour venu, sa maison se remplit comme jamais de clients. La nièce, que Lessa empêche depuis longtemps de rejoindre une congrégation pentecôtiste, s'inquiète d'un éventuel changement de disposition de sa tante. La réplique de cette dernière, mêlant scrupules de bonne professionnelle et conscience de la nécessité du travail, ne manque pas de bon sens : " Je ne renverrai pas mes clients parce que j'ai besoin de gagner ma vie. " La visite à l'Église quadrangulaire est alors reportée au soir.

Mais, dit Lessa, Satan n'entend pas être trompé de la sorte. Agissant comme un caboclo désireux de châtier un médium récalcitrant, il lui décoche une "flèche " invisible qui se fiche dans sa colonne vertébrale, l'empêchant de respirer et de parler. Les soins qu'on lui porte sont incapables de soulager la douleur. Elle refuse néanmoins de suivre Cecilia, arrivée sur ces entrefaites, qui ne s'étonne pas outre mesure de la tournure prise par les événements : "Je t'avais prévenue. Satan est traître."

Après une journée de travail particulièrement pénible, vers les sept heures du soir, Lessa jette pêle-mêle tous les objets du culte dans un grand sac qu'elle prétend remettre au pasteur. Son premier contact avec l'Église se fait dans la douleur physique et morale : elle souffre du dos, sa fille pleure et elle supporte le poids de l'énorme sac.

"Je suis restée toute tordue, je ne pouvais plus me tenir droite. Mais je te dis une chose, j'ai aussi été très en colère. J'ai pris qưelques sacs, j'ai pris les têtes [des statues de caboclos] et je les ai jetées dans le sac. Et j'ai coupé tout de suite les têtes, et j'ai jeté la faixa [écharpe portant le signe distinctif d'un caboclo], les guias [les colliers rituels] et les vêtements, tout ce qui appartenait aux démons. Tout, tu sais ce que c'est ? Tout.

15. Après Bahia, c'est l'État le plus prestigieux aux yeux des médiums de Belém, celui où l'on trouve les chefs de culte les plus respectueux des traditions africaines. 
J'étais très fâchée parce que l'homme des chemins [c'est-à-dire exu] m'a beaucoup battue. Ma fille souffrait aussi de cette influence, et elle n'a pas arrêté de pleurer comme une désespérée jusqu'à l'église. Et quand on est arrivé à l'église, le désespoir de ma fille m'a atteinte. J'ai dû descendre de l'autobus et prendre un taxi parce que je n'en pouvais plus. La douleur augmentait chaque fois plus. J'étais tordue, ma fille criait, et avec les bagages, on ne pouvait plus rester dans l'autobus. Nous avons tout emporté : vêtements, statues, tout. La seule évangélisation que j'ai eue, c'est celle-là, collègue. "

Arrivée à l'église, elle confie le sac au pasteur qui, avec un commentaire ironique, en sort un à un les objets des caboclos pour les montrer à l'assemblée. Bien que Lessa soit venue de son plein gré, elle ressent un tel geste comme une offense insupportable et sent inexorablement la colère monter en elle. Laissant alors les esprits s'emparer de son être et de sa conscience, elle entre en transe.

"J'ai donné ce lourd sac au pasteur, au pupitre. Il a commencé à distribuer tous ces vêtements et disait : "Regardez, ça c'est au caboclo Rompe-Mato, ça c'est à un autre..." Mais si tu savais, j'ai ressenti un désespoir dans l'âme, une envie de crier, de me jeter sur ce pasteur, de le dévorer, tu sais. Alors j'ai commencé à être désespérée, et tout de suite le diable s'est emparé de ma vie, là, à l'intérieur de l'église. Alors j'ai sombré dans l'inconscience. Ma nièce m'a raconté, il parât que je ressemblais à une sorcière. Elle dit que je criais, que je hurlais, et qu'il y avait deux pasteurs et un groupe d'abreiros ${ }^{16}$ autour de moi qui ont prié jusqu'à ce que le diable soit détruit. Ils ont demandé à Jésus de le brûler et, après, ils m’ont demandé si je me sentais bien. Et la flèche est sortie de mon dos. Et j'ai pris cette décision d'accepter Jésus, même s'ils ne m’ont rien demandé ce jour là."

Revenue à elle, Lessa éprouve un grand soulagement : grâce aux prières des pasteurs, la flèche est sortie de son dos et le diable a été "détruit ». Mais elle ne situe pas ce jour-là sa "conversion" au pentecôtisme. Cette possession démoniaque est la première d'une longue série à l'issue de laquelle Lessa pourra se dire "libérée " de l'influence des caboclos et du diable. L'exorcisme se déroulera dans une autre église de la même dénomination, plus proche de son domicile, et il sera mené à son terme par un jeune pasteur de vingt-quatre ans pour lequel Lessa ne cache pas son admiration. Remarquablement fervente, elle promet d'assister au culte deux fois par semaine; elle s'y rend en réalité tous les soirs.

Lessa aurait trouvé une alliée en la personne de sa nièce pour faire accepter sa nouvelle appartenance religieuse à son entourage. En effet, insiste-t-elle, ses faits et gestes pendant la possession lui sont rapportés par sa nièce ; ce qui lui permet d'affirmer par contrecoup que la transe est bien réelle, qu'il ne s'agit pas d'une affabulation de sa part ou d'une façon de se montrer "supérieure " aux autres (un comportement réprouvé qui prête aux commérages). Ce procédé narratif, qui introduit des témoignages de tiers comme preuves de l'inexorabilité d'un destin, n'est évidemment pas propre aux croyants. Le récit des médiums, après avoir insisté sur les moqueries dont ils étaient l'objet au début de leur carrière dans les cultes, tend toujours à faire endosser aux parents et voisins proches un rôle actif dans leur décision de se consacrer aux caboclos. Lessa, qui lors de son passage dans

16. Les obreiros sont les employés de l'église qui secondent le pasteur à l'heure du culte. 
les cultes n'a pas réussi à se constituer un tel cercle, semble avoir davantage de succès quand elle s'approche du pentecôtisme.

Il faut dire que le lien avec les êtres invisibles, si problématique dans les cultes qu'il est remis en question à chaque possession, est d'emblée accepté par les pentecôtistes qui travaillent à arracher l'être humain des griffes du démon. L'exorcisme pentecôtiste, qui ne distingue pas la filière de la possession de la filière thérapeutique ${ }^{17}$, traite en effet d'une façon semblable ce qui demeure toujours séparé dans les cultes. L'alliance avec les caboclos et l'agression par les esprits de l'encosto sont tenues dans les églises pour des formes différentes d'une même manifestation diabolique. La transe possessive, dans la mesure où elle révèle l'emprise des démons, constitue ainsi le premier pas vers la "libération par le Saint-Esprit ".

Or Lessa, qui n'est pas arrivée à faire de son boto autre chose qu'un encosto, est un bon sujet pour l'exorcisme pentecôtiste. Elle connaît les cultes, elle est capable de donner à voir une possession. Mais cette médium peu brillante, placée d'office au centre des attentions du pasteur et de ses assistants, n'a plus à être convaincante, pas plus qu'elle ne doit s'imposer dans la ronde des fils-de-saint ${ }^{18}$. À l'abri de toute concurrence, elle trouve au sein de l'Église une place qui lui est reconnue sans difficulté, où elle incarne le Mal combattu par les pentecôtistes.

"Ça va faire quatre ans que je me suis convertie. J'ai donné beaucoup de travail au pasteur parce que tous les soirs j'arrivais là angoissée et prostrée. Quand mon époux a su que j'avais emporté les choses [du culte], il mourait de peur qu'il arrive les pires choses chez nous. Je ne me disputais pas avec lui parce que j'acceptais tout. Mais il m'injuriait jour et nuit, il m'injuriait vraiment. Seulement j'avais reçu des conseils très précieux des pasteurs. Je ne devais rien répondre à ce qu'il me dirait. Car cela allait se produire. Alors tout ce qu'il disait entrait par une oreille et ressortait par l'autre. Je ne faisais que prier. J'ai promis d'aller au culte les mardis et les vendredis avec l'idée de ne plus jamais arrêter. Morale de l'histoire : tous les soirs j'allais à l'église et tous les soirs je tombais. J'étais accompagnée par des légions de démons qu'ils brûlaient au nom de Jésus, et ces choses malignes sont sorties de mon corps."

Les membres de la hiérarchie de l'Église l'ont prévenue de la réaction violente de Pedro. De fait, son compagnon, redoutant les représailles des caboclos, l'insulte et lui mène la vie dure. Faisant la sourde oreille comme les pasteurs le lui ont conseillé, Lessa s'abstient de jeter de l'huile sur feu par un commentaire acerbe. Chacun des conjoints fait appel à de nombreuses ruses pour faire changer l'autre d'avis. Pedro la tente parfois avec de la nourriture alors qu'elle jeûne; il essaie le plus souvent de faire valoir son autorité de chef de famille. Lessa est plus subtile. Au milieu du jeûne et des prières, elle glisse des éléments de chantage affectif: "Accepte Jésus si tu m'aimes et si tu veux ma libération. " Elle en appelle également à la fierté paternelle en suggérant à sa fille de convier son père à la voir chanter " devant le pupitre avec le microphone ". Enfin, à la saison des pluies, les orages torrentiels fournissent à Lessa un bon prétexte pour se faire conduire à

17. L'adorcisme et l'exorcisme dont parle Jean-Pierre Olivier de Sardan (1994) à propos du cas songhayzarma du Niger occidental.

18. Pour une description de l'exorcisme pentecôtiste, voir V. Boyer (1996a). 
l'église en voiture, sachant bien que Pedro ne supportera longtemps d'attendre inconfortablement installé dans le véhicule et qu'il finira par entrer dans le temple. Elle remporte une nouvelle bataille quand, en présence de son mari, le pasteur vient chercher à son domicile la raison de la persistance de ses possessions. Entrant dans la chambre du couple, celui-ci aperçoit, posé à côté de leur lit, un crucifix "adoré " par Pedro. Accédant au souhait pressant de sa femme, ce dernier consent à ce que le pasteur brise ce symbole de l'idolâtrie catholique. Lessa tombe aussitôt possédée. Les deux hommes l'emmènent dans cet état à l'église, où le "diable a tenté en vain de se faire passer pour un ange de lumière ". Cette expulsion conduite par le pasteur met un terme aux transes de Lessa et consacre sa libération.

"Avec tout ça j’ai vaincu au nom de Jésus » conclut-elle. Un dimanche, où comme à l'accoutumée le pasteur invite à s'avancer ceux qui désirent recevoir le baptême, Lessa se lève et marche jusqu'à l'estrade tout en "surveillant Pedro du coin de l'œil ». Voyant que son mari, sa nièce et sa fille l'ont suivie, elle connâit le baptême de feu par le Saint-Esprit descendu sur elle : elle se met ainsi à parler en "langues étranges». Quelque temps plus tard, le baptême par immersion confirmera officiellement l'intégration de toute la famille à la congrégation ${ }^{19}$.

\section{Au bout du compte, le succès}

Le statut actuel de Lessa (présidente du cercle des femmes) est directement lié à son itinéraire religieux. Car le temps passé dans les cultes de possession, bien qu'il se soit soldé par un échec et qu'il apparaisse rétrospectivement comme un séjour dans l'antre du diable, lui a permis d'acquérir un savoir sur les terreiros qui la qualifie pour le poste qu'elle occupe à présent ${ }^{20}$. Son expérience dans les cultes de possession et son vécu personnel de l'exorcisme la destinent ainsi presque naturellement, aux yeux des membres de sa congrégation, à assumer une fonction délicate, celle de "libérer » à son tour les médiums dont elle a partagé les certitudes. Ces séances lui procurent, semble-t-il, un vrai plaisir quelque peu inspiré par la revanche.

"Mercredi et vendredi, nous travaillons à la libération. Ces jours-là viennent des macumbeiros [autre nom des médiums], des personnes perturbées par l'esprit malin. Nous avons le plaisir de poser la main [sur leur tête] et de l'insulter. Insulter, ça veut dire défier cet esprit. Et l'esprit se met en colère et il se manifeste, au nom de Jésus. Et nous lui ordonnons qu'il tombe par terre, qu'il reste à genoux. Les caboclos sont interviewés. Le pasteur leur met le microphone devant la bouche pour qu'ils disent pourquoi ils sont en train de tourmenter cette vie, s'ils sont envoyés [par le diable] ou si

19. B. Jules-Rosette (1976) évoquait, à propos d'une Église africaine (les Apôtres de John Maranke), un processus de "conversion " par étapes qu'illustre également l'intégration progressive de Lessa à l'univers pentecôtiste.

20. D'autres expériences, définies comme déviantes par les pentecôtistes, peuvent également conférer une compétence négociable par le nouveau croyant. Ainsi, afin que ressorte sa compréhension du monde et de ses tentations, un pasteur me confiait son passé d'homosexuel et de prostitué. Un jeune garçon d'une autre Église ne montrait pas plus de réticence quand il s'étendait sur sa participation à des gangs liés au trafic de drogue. 
cette personne est macumbeira. Et après l'interview, nous demandons le feu, l'esprit est brûlé et la personne est libérée au nom de Jésus. C’est une expérience que j’ai depuis que j'ai accepté Jésus, une très bonne expérience. Le guide, le caboclo, l'esprit malin est brûlé et la personne devient normale, libre. Le feu saint vient pour brûler ce qui n'est pas bon dans notre corps, il brûle seulement les choses négatives. "

Dans cette description de l'exorcisme, il est clair que Lessa n'émet pas plus de réserve sur la réalité des caboclos que sur l'existence des démons. Qu'elle utilise indifféremment un mot ou l'autre montre assez bien que les esprits des cultes en viennent à se fondre sans difficulté dans la catégorie pentecôtiste des démons ${ }^{21}$. Lors de l'exorcisme, les esprits-démons sont convoqués d'une manière analogue aux caboclos pendant les cérémonies des terreiros. Mais alors que les esprits incarnés par les fils-de-saint dans les cultes reçoivent l'attention familière du public, les démons qui assaillent des membres de l'assistance dans une église sont maltraités. Le défi, l'insulte et la moquerie dont ils sont l'objet sont censés les affaiblir et les rendre inoffensifs pour faciliter leur expulsion par le pasteur et ses assistants ${ }^{22}$.

L'exorcisme pentecôtiste ne fournit assurément pas uniquement aux anciens fils-de-saint un cadre approprié pour reformuler une expérience religieuse antérieure, pas plus que les esprits des cultes n'épuisent le groupe des démons. Ainsi de nombreux pentecôtistes, qui se déclarent avoir été catholiques, soutiennent n'avoir eu aucun contact avec les terreiros, affirmation que vient cependant parfois nuancer une allusion à un parent ou un voisin médium dans leur entourage proche. Dans ce cas, le récit de leur parcours comporte également la mention d'un phénomène de possession qui aurait révélé l'emprise du démon sur eux, un démon qui ne se nommera jamais. Décrites comme des " absences où la personne tombe morte sur le sol ", ces possessions n'ont plus rien à voir avec le jeu élaboré des médiums des cultes. Enfin, le terme possession abandonne parfois toute référence à la transe possessive et prend un sens nettement métaphorique, quand les pentecôtistes, essentiellement des hommes, parlent par exemple du " démon »ou du « vice de l'alcool " qui les entravaient.

L'entrée dans le pentecôtisme s'accompagnerait, à en croire les membres de ce mouvement, d'un regard nouveau sur les relations entre les êtres humains. Ainsi, depuis que la présence du Saint-Esprit la protège comme un bouclier des diffi-

21. Lionel Caplan (1994), dans un article sur la croissance du néo-pentecôtisme dans le sud de l'Inde, fait une remarque semblable. L'auteur observe que l'insistance des pentecôtistes sur les forces du mal facilite l'absorption de la catégorie des peey, regroupant des êtres d'origine humaine ou non susceptibles de posséder les vivants. Les pratiques pentecôtistes seraient alors vues comme une " justification bịblique authentique" (ibid. : 124) des croyances de l'hindouisme local, ce qui expliquerait en partie le succès remporté par ce mouvement auprès des classes populaires urbaines.

22. D'une certaine façon cette mise en scène évoque un contexte très différent et éloigné du pentecôtisme, celui de l'exorcisme sri lankais étudié par Bruce Kapferer (1991 : 316) où les démons " are treated as if they were gods". Cependant toutes les offrandes, "which seem appropriate to the gods [...] carry the idea of the polluting and destructive quality of the demons : the incense has the smell of death and pollution, the flowers are shredded [and] the foods are cooked and mixed with pollutants such as oil, flesh and blood. But the demons in their acceptance of them become vulnerable to their own destruction ". Dans le pentecôtisme brésilien également, les démons, provoqués à montrer leur force, sont censés aller à leur perte. 
cultés de l'existence, Lessa peut affirmer : « Nous arrivons à supporter une offense, une calomnie, nous savons prier pour celui qui nous a calomniés, nous savons pardonner, aimer notre prochain avec tous ses défauts. "Car la vie de péché, héritée d'Adam, cesse d'être le lot de ceux qui ont accepté Jésus, même s'il leur reste la chair et son cortège de tentations. Le jeûne et les prières, qui « vivifient et élèvent l'esprit ", permettent cependant de la mortifier et d' "étouffer " ses cris.

Ce n'est pas pour autant que Lessa se croit supérieure aux êtres humains qu'elle côtoie. Elle se sait encore " pécheresse " et "faillible", et surtout elle ne se montrerait pas aussi soumise à son mari qu'il sied à une femme pentecôtiste. La vie qu'elle a menée, son indépendance tôt conquise vis-à-vis de son père, son autonomie financière, sa liberté chèrement défendue expliqueraient aujourd'hui ses difficultés à se conformer aux commandements bibliques. Pedro, à qui elle a montré le chemin mais devenu depuis "plus croyant qu'elle ", ne manque pas de la chapitrer à ce sujet. Lessa s'estime pourtant satisfaite "pour le peu de temps qu'elle a passé dans la main de Dieu, car elle a eu une vie plus longue en compagnie de Satan qu'avec Dieu ». Il n'est d'ailleurs pas dit qu'elle se soumette de si tôt. En effet, à force d'intercéder pour ses proches, d'exorciser les diables des autres et de lutter contre Satan, elle oublie, dit-elle, de prier " pour que sa vie soit transformée " et, par là même, de s'en prendre à ses propres démons.

Ce qu'elle désigne comme son principal défaut la lie à son insu à l'univers des cultes de possession, ou plutôt au dispositif qui y régule les relations entre hommes et femmes. Dans les cultes, le façonnage du personnage du caboclo permet à chaque femme médium de dicter ses volontés à son entourage et à son compagnon, tout en niant se départir d'une attitude de réserve. L'alternative pentecôtiste consiste à modeler l'homme de chair et de sang, à l'encourager à s'engager dans la carrière de pasteur et à le contraindre à renoncer à l'attrait de la rue. Le changement souhaité par Lessa ne surviendra d'une certaine façon que si elle réussit là où elle a échoué dans les cultes, à savoir dans la manipulation selon des critères féminins d'une autorité masculine. À l'ombre d'un homme qui ne lui échappera pas, puisqu'ils sont tous deux « liés devant Dieu et par lui », elle pourra étendre son influence sur le groupe.

Le récit de Lessa, qui se dit aujourd'hui " pentecôtiste convaincue ", rejoint, dans ses grandes lignes comme dans de nombreux détails, le tableau dressé par des femmes restées pour leur part dans les cultes de possession. Parmi les thèmes récurrents, la place attribuée au compagnon de ces femmes et l'extériorité de l'origine du mal sont parmi les plus évidents. Car l'homme, et le Mal qui survient par les autres, sont les fils narrateurs utilisés pour construire un rôle bien particulier : celui de victime. Que les esprits des cultes deviennent des démons dans le récit pentecôtiste ne modifie en rien le dispositif narratif. L'attitude adoptée par les femmes qui jettent un regard semblable sur la société, et les événements qu'elles retracent, leur permettent en effet de définir, au-delà d'une appartenance religieuse spécifique, une position qui leur reviendrait dans les relations sociales et les groupes domestiques. C'est de cette position, revendiquée haut et fort, qu'elles agissent sur leur entourage. 
La définition d'une orthodoxie religieuse, par l'indication de ce qui est "bon et bien ", n'a pas seulement des visées stratégiques et des effets de partition du champ religieux. Elle est également supposée avoir des conséquences éthiques, en fournissant aux membres des Églises des normes et des valeurs qui désormais doivent gouverner leur existence : l'amour de Dieu devient le modèle de toute relation entre les êtres humains, le respect des commandements bibliques est érigé en règle de vie, la crainte du péché opère comme une contrainte personnelle.

Or, Anthony P. Cohen (1994: 129-130) a fort bien analysé l'enjeu des limites en montrant qu'elles sont d'autant plus fortement investies symboliquement par la société qu'elles sont ambiguës pour l'individu : "If we recognize boundaries as matters of consciousness rather than institutional dictation, we see them as being rather more amorphous and ambiguous than we otherwise have done. It may be this ambiguity which inclines societies to invest their various boundaries so heavily with symbolism [...] As a matter of ideology, the boundary may be given dogmatic form. But its internalisation in the consciousness of individual renders it much less definitely. "

Les pasteurs, qui serinent à leurs ouailles la nécessité de se garder des tentations, ne permettent à aucun croyant d'ignorer que certaines pratiques religieuses ou certains comportements leur font franchir les limites du Bien. Toutefois, il n'est pas sûr que les notions fondant l'établissement de ces frontières aient la même pertinence et soient entendues de la même façon par les membres des Églises pris séparément. Le cas de Lessa illustre parfaitement cette idée. En effet, en considérant ses "écarts de comportement " avec quelque indulgence, cette femme reste plus proche d'une conception du Mal en termes d'une "faiblesse tolérable" (ce que ne démentiraient pas les médiums des cultes) que comme un "pouvoir à maîtriser" (ce qui reflète l'interprétation pentecôtiste habituelle) ${ }^{23}$. Elle se trouve plus à son affaire dans la flexibilité et le relatif que dans l'absolu et le rigorisme. Ainsi que l'écrit Robert W. Hefner (1993 : 15), il faut distinguer " cultural rationalization - the enunciation, systematization, and formalization of cultural truths in light of a particular value or ideal, a quality of sociocultural systems - from the broader concept of rationality, or the effectiveness of certain ideas at making sense of an individual or group's life-world, again with reference to some underlying value complex ».

La narration de Lessa, tout comme la conduite qu'elle nous rapporte, sont plus construites en fonction de la conscience qu'elle a d'elle-même et de sa relation avec l'entourage social ${ }^{24}$ - laquelle est sujette à des variations et à des réajustements successifs - que par la référence à un principe unique organisant son récit, fût-il celui de l'orthodoxie pentecôtiste. Il convient de souligner la créativité et l'ingéniosité de Lessa, au-delà de la condition commune qu'elle partage avec les membres des classes populaires urbaines, pour constituer l'idiosyncrasie de son récit. Celui-ci est tout d'abord imprégné de formes d'interprétation des événements qui sont propres aux

23. Cultes de possession et Églises pentecôtistes semblent illustrer parfaitement la formulation de David Parkin (1994:9) sur les deux sens du Mal.

24. Pour reprendre ici encore les termes de A. P. Cohen (1994: 168). 
cultes de possession. Le recours narratif à l'intervention de figures invisibles dans l'existence des êtres humains, pour expliquer leurs rivalités, justifier de choix personnels ou même de l'acquisition de biens matériels, compte parmi les plus importants.

Mais ce récit reflète aussi le vécu personnel d'une expérience concernant une bonne partie de la population : la trajectoire de migration de Lessa. C'est ainsi qu'elle intègre des éléments plus spécifiquement liés au milieu rural dont elle est originaire. À la différence des médiums qui s'emparent de l'un des nombreux caboclos invisibles, personnages anthropomorphiques peuplant l'univers des terreiros, pour en faire leur double dans la possession, Lessa tente d'étoffer sa représentation d'une figure zoomorphique, le boto, dont les aventures se rapportent directement à l'imaginaire amazonien rural. Or, si les fils-de-saint des cultes de possession urbains admettent que leurs caboclos ont bien souvent une forme animale, ils croient aussi que leur efficacité est plus grande quand ils se présentent sous une apparence humaine. Lessa, incapable de donner un nom de caboclo et des traits de comportements humains à son boto, ne saura donc pas le rendre opérationnel dans des cultes urbains. Dans un autre registre, elle ne parviendra pas à exploiter durablement l'étroitesse des liens entre migrants.

C'est dans le giron pentecôtiste qu'enfin son expérience personnelle se construit comme identité. En effet, son appartenance présente (ce " new locus of self-definition " dont parle Robert W. Hefner (ibid. : 17)) tolère des approximations et des erreurs d'évaluation qui ont été durement sanctionnées dans les cultes de possession. Lessa peut donc enrichir son récit de multiples références et de divers emprunts qui comblent la pauvreté mythologique des croyances pentecôtistes à proprement parler. Car peu resterait à dire une fois reconnu que Jésus est l'unique sauveur et que le Saint-Esprit répand encore aujourd'hui ses dons sur les hommes. Cela est si vrai que le raisonnement permettant de parachever cette identité pentecôtiste, c'est-à-dire l'antériorité de l'élection de Lessa par Jésus sur celle par le boto, provient d'un autre système religieux.

Si les membres du mouvement évangélique affirment détenir la seule vérité qui soit, protégés qu'ils seraient par Jésus, ils ne revendiquent pas ouvertement pour l'ensemble de leurs pratiques et croyances le statut de "vraie religion". Lusage du terme religion, pour désigner les croyances pentecôtistes et celles des autres, supposerait reconnaître implicitement une certaine égalité entre les différents systèmes religieux, et le jeu des relations de concurrence. Or la prétention à l'universalité du mouvement évangélique va de pair avec la réduction des autres systèmes religieux à des formes locales et limitées dans leur capacité d'explication. $\mathrm{La}$ " conversion ", dont les membres des Églises ne cessent de parler, signifie pour eux accéder à des notions qu'on peut dire " totalitaires " dans la mesure où elles sont censées pouvoir recouvrir les autres systèmes, et donc vaincre les forces des ténèbres et du Mal qui maintiennent, au mieux, les hommes dans un état de savoir parcellaire. Le pentecôtisme ne s'affirme pas comme une autre manière de voir le monde mais comme la seule manière de l'appréhender dans sa totalité.

MOTS CLÉS : cultes de possession - pentecôtisme - conversion - Brésil. 
Bougerol, Christiane

1993 "Le cumul magico-religieux à la Guadeloupe ", Journal de la Société des Américanistes 79 : 91-103.

Boyer, Véronique

1992 « De la campagne à la ville : la migration du caboclo", Cabiers d'Études africaines XXXII (1), 125 : 109-127.

1993 Femmes et cultes de possession au Brésil : les compagnons invisibles. Paris, L'Harmattan.

1996a " Possession et exorcisme dans une Église pentecôtiste au Brésil ", Cahiers des Sciences humaines 32, 2 : 243-264. Paris, ORSTOM.

$1996 b$ "Le don et l'initiation. De l'impact de la littérature sur les cultes de possession au Brésil ", L'Homme 138 : 7-24.

Caplan, Lionel

1994 "The Popular Culture of Evil in Urban Sourh India ", in David Parkin, ed., The Anthropology of Evil. Cambridge, Blackwell.

Cohen, Anthony P.

1994 Self-Consciousness : An Alternative Anthropology of Identity. London, Routledge.

Favret-Saada, Jeanne

1985 Les mots, la mort, les sorts. La sorcellerie dans le bocage. Paris, Gallimard ("Folio "). (1 re éd. 1977.)

Fonseca, Claudia

1984 "La violence et la rumeur : le code d'honneur dans un bidonville brésilien ", Les Temps modernes 455 : 2193-2235.

1988 "Alliés et ennemis en famille ", Les Temps modernes 499 : 28-58.
Galvāo, Eduardo

1976 Santos e visagens : um estudo da vida religiosa de Itá, Baixo Amazonas. São Paulo,

Hefner, Robert W.

1993 "Introduction : World Building and the Rationality of Conversion ", in Robert W. Hefner, ed., Conversion to Christianity. Berkeley, University of California Press.

Jules-Rosette, Benetta

1976 "The Conversion Experience : The Apostles of John Maranke ", Journal of Religion in Africa VII, 2 : 132-164.

Kapferer, Bruce

1991 A Celebration of Demons : Exorcism and the Esthetics of Healing in Sri Lanka. Oxford, Berg/Washington DC, Smithsonian Institution Press.

Olivier de Sardan, Jean-Pierre 1994 "Possession, affliction et folie : les ruses de la thérapisation ", L'Homme 131 : 7-27.

Parkin, David 1994 "Introduction ", in David Parkin, ed., The Anthropology of Evil. Cambridge, Blackwell.

Wagley, Charles

1977 Uma comunidade amazônica. Estudo do homem nos trópicos. São Paulo, Companhia Editora Nacional.

Zaluar, Alba

1982 "As mulheres e a direção do consumo doméstico : estudo de papéis familiares nas classes populares urbanas ", Cocha de Retalhos: Estudos sobre a familia no Brasil. São Paulo, Brasiliense : 161-184.

Zempléni, András

1975 "De la persécution à la culpabilité ", in Colette Piault, ed., Prophétisme et thérapeutique: Albert Atcho et la communauté de Bregbo. Paris, Hermann ("Savoir"). 